\title{
Nuclear polyadenylation factors recognize cytoplasmic polyadenylation elements
}

\author{
Andrea Bilger, Catherine A. Fox, ${ }^{1}$ Elmar Wahle, $^{2}$ and Marvin Wickens ${ }^{3}$ \\ Department of Biochemistry, College of Agriculture and Life Sciences, University of Wisconsin, Madison, Wisconsin \\ 53706 USA; ${ }^{2}$ Biozentrum, University of Basel, Basel, Switzerland
}

In the cytoplasm of oocytes and early embryos, addition of poly(A) to mRNAs can activate their translation. We demonstrate that despite many differences between poly $(A)$ addition in the cytoplasm and nucleus, these two forms of polyadenylation may involve identical trans-acting factors. Nuclear polyadenylation requires the sequence AAUAAA, the AAUAAA-binding cleavage and polyadenylation specificity factor (CPSF), and a poly(A) polymerase (PAP). We show that CPSF and PAP, purified from calf thymus, exhibit the same sequence specificity observed in the cytoplasm during frog oocyte maturation, requiring both AAUAAA and a proximal U-rich sequence. The enhanced polyadenylation of RNAs containing U-rich sequences is caused by their increased affinity for CPSF. Frog nuclear polyadenylation factors display cytoplasmic sequence specificity when dilute, suggesting that a difference in their concentrations in the nucleus and cytoplasm underlies the different sequence specificities in the two compartments. Because polyadenylation in extracts prepared from oocytes before maturation is stimulated by addition of CPSF, the onset of polyadenylation during early development may be attributable to the activation or synthesis of a CPSF-like factor. We suggest that sequences upstream of AAUAAA that are required for cleavage and polyadenylation of certain pre-mRNAs in the nucleus may be functionally equivalent to the upstream, U-rich sequences that function in the cytoplasm, enhancing CPSF binding. We propose that CPSF and PAP comprise a core polyadenylation apparatus in the cytoplasm of oocytes and early embryos.

[Key Words: Poly(A); early development; translational control]

Received February 14, 1994; revised version accepted March 18, 1994.

Most modifications of mRNA precursors, including capping, splicing, and $3^{\prime}$ - end cleavage, occur exclusively in the nucleus. Of the many covalent modifications that mRNAs undergo, only the addition of poly(A) to the $3^{\prime}$ ends of messages is known to occur in both the nucleus and the cytoplasm. During early development in many animal species, cytoplasmic addition of poly(A) can control translation of specific maternal mRNAs. Certain mRNAs receive additional poly $(A)$ and become translationally active, whereas others lose their tails and cease translation (for review, see Jackson and Standart 1990; Wickens 1992). The mechanisms of cytoplasmic poly(A) addition and of its effects on translation present key problems in both RNA processing and early development.

Polyadenylation in the nucleus and in the cytoplasm differ substantially. Nuclear polyadenylation appears to be constitutive and adds 200-300 adenosines to virtually every mRNA (for review, see Wahle and Keller 1992). In contrast, cytoplasmic polyadenylation is highly regulated: mRNAs receive poly(A) of different lengths and at

Present addresses: ${ }^{1}$ Department of Molecular and Cellular Biology, University of California, Berkeley, California 94721 USA.

${ }^{3}$ Corresponding author. different times during development (e.g., Paris and Philippe 1990; Sheets et al. 1994; for review, see Dworkin and Dworkin-Rastl 1990; Wickens 1990). Furthermore, whereas nuclear polyadenylation is coupled to cleavage of the pre-mRNA, cytoplasmic polyadenylation is not. Rather, the same mRNAs that receive poly(A) in the cytoplasm have already undergone cleavage and polyadenylation in the nucleus. Once they enter the cytoplasm, they are shortened by a cytoplasmic poly(A) removal activity (Huarte et al. 1992). As a result, the substrates for cytoplasmic polyadenylation already possess short tails; the substrates for nuclear polyadenylation do not.

Nuclear polyadenylation requires the highly conserved sequence AAUAAA, as well as several trans-acting protein factors (for review, see Wahle and Keller 1992). The AAUAAA sequence is located 5-35 nucleotides upstream of the nucleotide to which poly(A) is added. AAUAAA interacts directly with the cleavage and polyadenylation specificity factor (CPSF), a complex of three or perhaps four proteins (Bienroth et al. 1991; Murthy and Manley 1992). Poly(A) polymerase (PAP) catalyzes the polymerization of ATP into poly(A) (Takagaki et al. 1988; Ryner et al. 1989; Bardwell et al. 1990; Wahle 1991a). Poly(A)-binding protein II (PABII) promotes processive elongation of the poly(A) tail (Wahle 1991b). 
AAUAAA appears to be the only specific sequence necessary for nuclear polyadenylation, in that AAUAAA-containing RNAs as short as 11 nucleotides interact with CPSF and receive poly $(\mathrm{A})$ in vitro (Wigley et al. 1990). Similarly, base modifications upstream and downstream of AAUAAA do not significantly impair nuclear polyadenylation or CPSF binding (Conway and Wickens 1987; Keller et al. 1991). The interaction between AAUAAA and CPSF is highly specific: It is prevented by single point mutations, base modifications, or ribose methylations within AAUAAA (Bardwell et al. 1991; Bienroth et al. 1991; Keller et al. 1991; Murthy and Manley 1992). These results, as well as those of gel retardation and UV cross-linking experiments (Bardwell et al. 1991; Bienroth et al. 1991; Keller et al. 1991), demonstrate unambiguously that CPSF binds directly to AAUAAA.

Cytoplasmic polyadenylation has been studied most extensively during early development, in the period termed oocyte maturation. During maturation, frog oocytes advance from first meiotic prophase to second meiotic metaphase, becoming eggs. Oocytes mature in vitro in response to progesterone. During maturation, the oocyte nucleus breaks down, permitting nuclear and cytoplasmic contents to mix. Polyadenylation during oocyte maturation requires two sequences in the mRNA: AAUAAA and a nearby U-rich sequence (e.g., UUUUUAU) (Fox et al. 1989; McGrew et al. 1989; Paris' and Richter 1990). Because virtually all mRNAs contain AAUAAA, the U-rich sequences-also termed cytoplasmic polyadenylation elements (CPEs)-are responsible for the specificity of cytoplasmic polyadenylation.

Whole-cell extracts of frog eggs support polyadenylation with the same sequence specificity seen during oocyte maturation (Paris and Richter 1990; Fox et al. 1992) and have been used to identify essential trans-acting factors. At least two chromatographic fractions derived from this extract-a PAP and an RNA-binding activity-are necessary to reconstitute the reaction in vitro (Fox et al. 1992). The RNA-binding fraction interacts specifically with RNAs containing both AAUAAA and a U-rich sequence, and confers a preference for both sequences on purified PAP (Fox et al. 1992). The RNAbinding fraction, and perhaps the binding activity itself, appears to be activated in response to progesterone (Fox et al. 1992). In unfractionated egg extracts, proteins of 58 and $82 \mathrm{kD}$ can be cross-linked by UV light to different CPE-containing mRNAs (McGrew and Richter 1990; Paris et al. 1991). Because cross-linking requires a CPE and AAUAAA in both cases, these proteins presumably are involved in polyadenylation during oocyte maturation.

The factors that catalyze polyadenylation during oocyte maturation are cytoplasmic. Manual removal of the nucleus from an oocyte does not impair its ability to add poly(A) during subsequent maturation (Fox et al. 1989). In addition, certain RNAs injected into the cytoplasm receive poly(A) before the nucleus has broken down (McGrew and Richter 1990; Paris and Richter 1990; Sheets et al. 1994).
Despite differences in cellular location, regulation, and sequence specificity, cytoplasmic and nuclear polyadenylation factors may be related. The PAP activities present in extracts of frog eggs and of calf thymus fractionate similarly (Fox et al. 1992). Furthermore, calf thymus PAP, when mixed with the frog egg RNA-binding fraction, becomes specific for RNAs containing AAUAAA and a U-rich element (Fox et al. 1992). Likewise, the stimulatory activity found in the egg RNAbinding fraction fractionates as does calf thymus CPSF through several chromatographic steps (Fox et al. 1992; C. Fox, A. Bilger, and M. Wickens, unpubl.). However, although CPSF appears to require only AAUAAA to bind to RNA and stimulate polyadenylation, the egg RNAbinding fraction requires both AAUAAA and a U-rich sequence (Fox et al. 1992).

In this report we have reconstituted the sequence specificity of cytoplasmic polyadenylation during early development using purified polyadenylation factors from somatic cells. CPSF and PAP purified from calf thymus, specifically polyadenylate RNAs containing both a CPE and AAUAAA, demonstrating a sequence specificity indistinguishable from that observed during frog oocyte maturation. We propose that CPSF, or a closely related molecule, imparts sequence specificity to cytoplasmic, as well as nuclear, polyadenylation, and that regulation of CPSF activity in the cytoplasm activates polyadenylation during early development. We suggest that competition for CPSF in the cytoplasm, but not in the nucleus, underlies the differences in the compartments. In the simplest hypothesis, the enzymes that add poly(A) in the nucleus and cytoplasm are the same.

\section{Results}

Purified calf thymus PAP and CPSF display maturation-specific polyadenylation activity

Biochemical fractionation studies suggested that the RNA-binding activity present in frog egg extracts might be similar to mammalian CPSF (Fox et al. 1992; C. Fox, A. Bilger, and M. Wickens, unpubl.|. Therefore, we tested whether purified CPSF could functionally substitute for the egg RNA-binding fraction and cause purified PAP to preferentially add poly(A) to substrates containing a U-rich element near AAUAAA.

We incubated purified calf thymus CPSF and PAP with radioactively labeled segments of the Xenopus c-mos 3'untranslated region ( $3^{\prime}$ UTR) transcribed in vitro. Poly(A) addition was assayed by gel electrophoresis and autoradiography. The c-mos substrates either contained UUUUAU, as does wild-type c-mos mRNA, or lacked it because of a 6-nucleotide substitution (Fig. 1A). Both substrates contained AAUAAA. In vivo, c-mos mRNA receives poly $(\mathrm{A})$ during maturation (Paris and Richter 1990; Sheets et al. 1994), in a reaction stimulated by its UUUUAU sequence (Paris and Richter 1990; C. Fox, M. Sheets, and M. Wickens, unpubl.). The UUUUAU sequence enhances polyadenylation by purified calf thymus CPSF and PAP (Fig. 1A, lanes 1-6). Stimulation by 
Bilger et al.

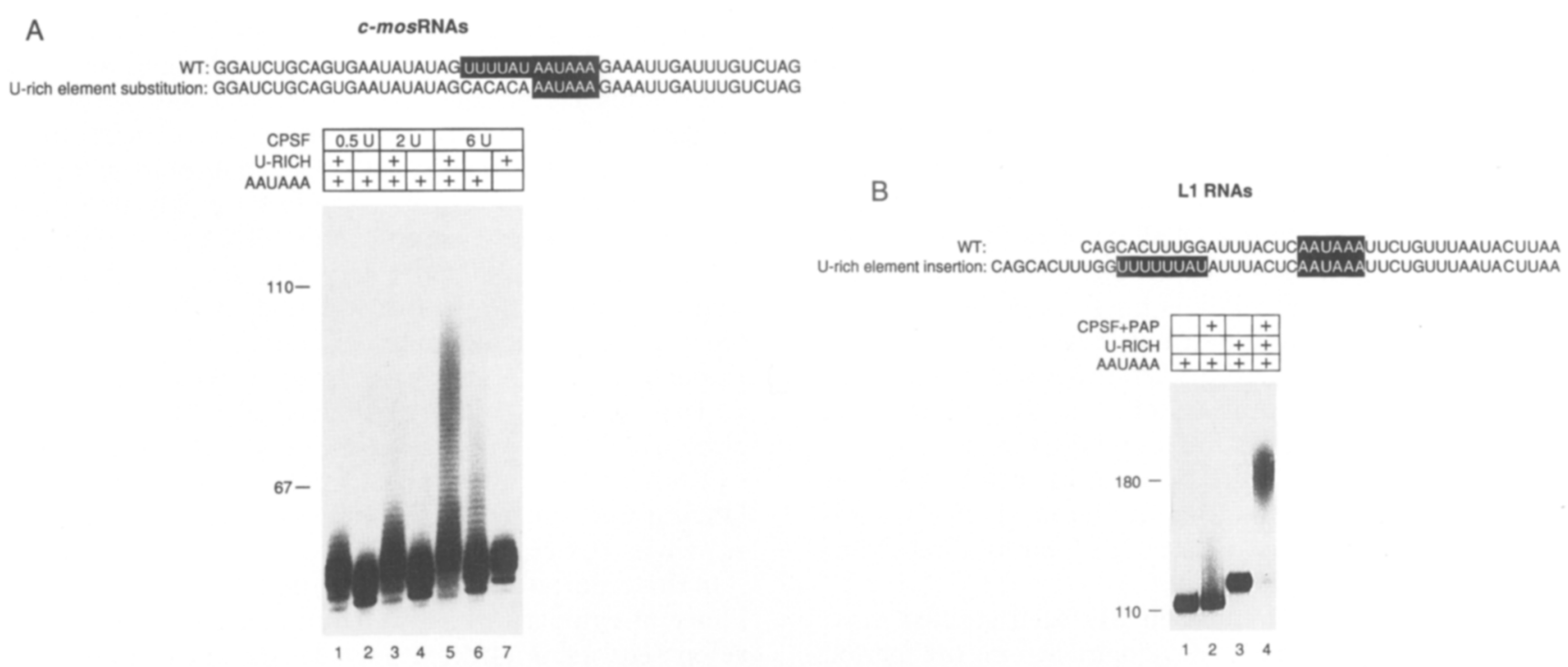

Figure 1. Purified calf thymus CPSF and PAP display maturation-specific polyadenylation activity. (A) The UUUUAU sequence in c-mos mRNA stimulates polyadenylation by purified CPSF and PAP. Substrates were derived from the last 50 nucleotides of $\mathrm{c}-\mathrm{mos}$ mRNA. RNAs were incubated with 25 units of pure PAP (units as defined by Fox et al. 1992) and the indicated amounts of CPSF (units defined by Bienroth et al. 1991) for $20 \mathrm{~min}$ at $25^{\circ} \mathrm{C}$. Incubation with 25 units PAP alone does not result in polyadenylation (not shown). (Lanes 1,3,5). Wild-type c-mos RNA; (Lanes 2,4,6) c-mos RNA containing AAUAAA but not UUUUAU; (lane 7) c-mos RNA containing UUUUAU but not AAUAAA (AAGAAA instead). (UUUUAU and AAUAAA are highlighted with black.) (B) Insertion of UUUUUUAU into L1 mRNA stimulates polyadenylation by CPSF and PAP. Substrates are derived from the last 104 nucleotides of L1 mRNA. RNAs were incubated with 12 units of CPSF and $\sim 150$ of units PAP for 20 min at $25^{\circ} \mathrm{C}$ (lanes 2,4). (Lanes 1,2 ) Wild-type L1 RNA; (lanes 3,4) L1 with UUUUUUAU. (UUUUUUAU and AAUAAA are highlighted with black.)

the UUUUAU sequence occurs throughout the range of CPSF concentrations tested. Polyadenylation increases progressively with the amount of CPSF added and requires AAUAAA (Fig. 1A, lane 7). We conclude that polyadenylation of the c-mos 3' UTR by purified CPSF and PAP is enhanced by the presence of a U-rich element near AAUAAA.

To confirm that U-rich elements stimulate polyadenylation by CPSF and PAP, we analyzed a substrate in which the sequence UUUUUUAU was inserted into a portion of the 3' UTR of ribosomal protein Ll mRNA (Fig. 1B). During maturation, this RNA receives poly(A), whereas wild-type L1 mRNA, lacking the U-rich sequence, does not (Varnum and Wormington 1990; S. Thompson and M. Wickens, unpubl.). Similarly, in vitro, the sequence UUUUUUAU dramatically enhances polyadenylation by purified CPSF and PAP (Fig. 1B). These data confirm that CPSF and PAP recognize a U-rich element upstream of AAUAAA, in addition to AAUAAA itself. This implies that CPSF or a related molecule may participate in cytoplasmic polyadenylation during maturation.

CPSF and PAP display cytoplasmic sequence specificity with a variety of substrates

To test further whether a CPSF-like factor is involved in polyadenylation during maturation, we analyzed three additional RNA substrates (Fig. 2). The $3^{\prime}$ UTRs of $\mathrm{H} 4$ and cyclin B1 RNAs, which receive poly(A) during mat- uration in vivo (Dworkin and Dworkin-Rastl 1985; Sheets et al. 1994), are polyadenylated in vitro by purified CPSF and PAP (Fig. 2). Xfin RNA, which does not

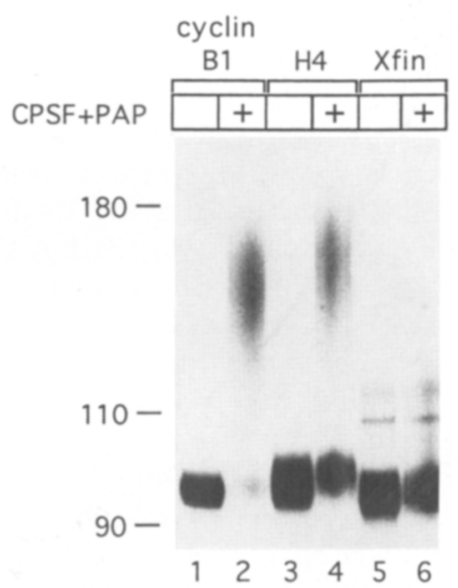

Figure 2. CPSF and PAP display cytoplasmic sequence specificity with a variety of substrates. Substrates are derived from the last $\sim 80$ nucleotides of the indicated RNAs /see Materials and methods). RNAs were incubated with $\sim 150$ units of PAP and 12 units of CPSF for $20 \mathrm{~min}$ at $25^{\circ} \mathrm{C}$. (Lane 1) Cyclin $\mathrm{B} 1$ RNA, no protein added; (lane 2) cyclin Bl RNA incubated with CPSF and PAP; (lane 3) H4 RNA, no protein added; (lane 4) H4 RNA incubated with CPSF and PAP; (lane 5) Xfin RNA, no protein added; (lane 6) Xfin RNA incubated with CPSF and PAP. H4 mRNA encodes a protein of unkown function; it is not histone $\mathrm{H} 4$ mRNA. 
receive poly $(\mathrm{A})$ during maturation, is not polyadenylated by CPSF and PAP (Fig. 2). Thus, for five substrates-cmos, L1, cyclin B1, H4, and Xfin-and mutants thereof, purified calf thymus CPSF and PAP accurately recapitulate the sequence specificity of cytoplasmic polyadenylation during oocyte maturation.

\section{CPSF stimulates an inactive oocyte extract}

Whole-cell extracts of matured oocytes, or eggs, efficiently polyadenylate mRNAs, whereas extracts of nonmature oocytes are much less active (Paris and Richter 1990; Fox et al. 1992). Polyadenylation in both extracts requires U-rich sequences in addition to AAUAAA. The relative lack of polyadenylation activity in oocyte extracts appears to be attributable to the lack of an RNAbinding activity: Addition of egg RNA-binding fraction to oocyte extract stimulates CPE-specific polyadenylation, whereas addition of egg PAP does not (Fox et al. 1992). If the key factor in the egg RNA-binding fraction is CPSF-like, as our results suggest, then addition of purified CPSF should also stimulate polyadenylation in the otherwise inactive oocyte extract. To test this prediction, we added purified calf thymus CPSF to an oocyte extract and assayed polyadenylation activity using the labeled c-mos RNAs described in Figure 1A.

Purified CPSF activates polyadenylation in the oocyte extract, in a concentration-dependent manner (Fig. 3). As expected, polyadenylation is stimulated by the presence of a U-rich element near AAUAAA and requires AAUAAA. These results suggest that a CPSF-like factor

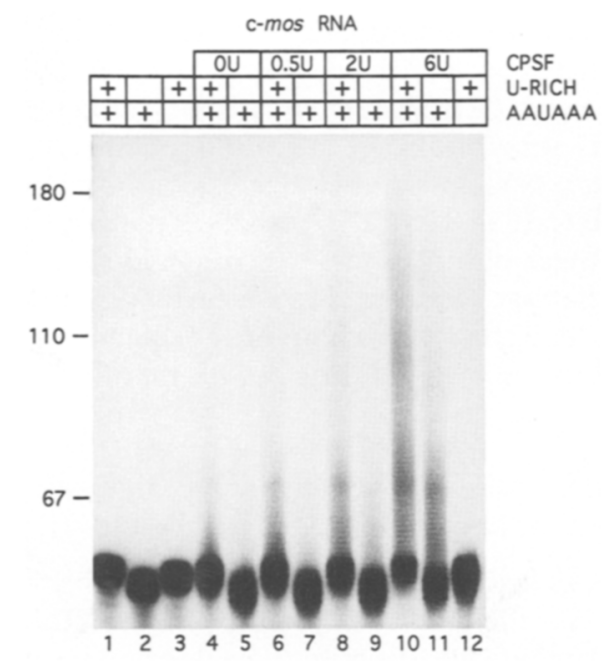

Figure 3. Purified calf thymus CPSF activates polyadenylation in an oocyte extract. Substrates are the c-mos RNAs shown in Fig. 1A. RNAs were incubated with $140 \mu \mathrm{g}$ crude oocyte extract 1 25 of units PAP activity) and the indicated amount of CPSF for $20 \mathrm{~min}$ at $25^{\circ} \mathrm{C}$. (Lanes $1,4,6,8,10$ ) Wild-type c-mos RNA; (lanes 2,5,7,9,11) c-mos RNA containing AAUAAA but not UUUUAU; (lanes 3,12) c-mos RNA containing UUUUAU but not AAUAAA. (Lanes 1,2,3) No protein added; (lanes 4,5) 0 units of CPSF; (lanes 6,7) 0.5 units of CPSF; (lanes 8,9) 2 units of CPSF; (lanes 10,11,12) 6 units of CPSF. limits polyadenylation in oocyte extracts, and imply that such a factor may be activated to turn on cytoplasmic polyadenylation during oocyte maturation.

\section{A U-rich element increases the affinity of CPSF for the substrate}

CPSF and PAP might preferentially add poly(A) to RNAs with U-rich sequences because CPSF binds more tightly to such RNAs. To test this notion directly, we determined the relative affinity of CPSF for RNAs with or without a U-rich element using a mobility shift assay (Fig. 4). Equal amounts of labeled L1 RNAs with or without UUUUUUAU were mixed together and incubated with various concentrations of CPSF. The mixture was then electrophoresed under nondenaturing conditions (Fig. 4A). RNAs that had bound to CPSF and RNAs that had not were recovered from the gel and analyzed by electrophoresis under denaturing conditions (Fig. 4B). Because the two RNAs differ in length by 8 nucleotides (UUUUUUAU), they migrated as two distinct bands in the denaturing gel and their relative abundances could be determined. CPSF preferentially interacts with the UUUUUUAU-containing RNA: That RNA is more abundant than Ll RNA in the CPSF complexes and correspondingly less abundant than Ll in the free RNA. As expected of a simple RNA-protein interaction, the difference in binding between the two RNAs is greatest at the lowest CPSF concentration; as the concentration of CPSF increases, more of each RNA enters the complex and the discrimination between the two RNAs decreases. Quantitative analysis of these data reveals that CPSF has approximately a fivefold greater affinity for the RNA containing UUUUUUAU.

\section{Specificity for U-rich elements is attributable to CPSF and PAP}

In the experiments presented thus far, both CPSF and PAP were purified from calf thymus. Although both factors are highly purified, neither is homogeneous (see below). Therefore, we examined whether the enhancement of polyadenylation by U-rich sequences was caused by CPSF and PAP, rather than by a contaminant present in either preparation.

To examine the PAP, the protein was overexpressed and purified from an Escherichia coli strain carrying a calf thymus PAP cDNA clone (Wahle et al. 1991; G. Martin and W. Keller, unpubl.). When combined with calf thymus CPSF, this recombinant PAP displayed the same preference for UUUUUUAU seen previously (not shown). These results demonstrate that PAP is sufficient to reconstitute U-rich specificity when mixed with CPSF.

Comparable experiments are not possible with CPSF, as that factor is not available in recombinant form. However, three lines of evidence argue that CPSF itself discriminates U-rich sequences. 
Figure 4. A U-rich element increases the affinity of CPSF for the substrate. (A) Radiolabeled L1 wild-type RNA and L1 RNA with a UUUUUUAU insertion were mixed together and incubated with the indicated amounts of CPSF. This mixture was then electrophoresed under nondenaturing conditions, transferred to nitrocellulose, and visualized by autoradiography. The positions of CPSF-RNA complexes and of free RNA (uncomplexed with protein) are indicated with brackets. (B) RNAs contained in the complex and free bands from $A$ were eluted from the nitrocellulose and analyzed by electrophoresis under denaturing conditions, followed by autoradiography. One fmole of this preparation of CPSF corresponds to $1.5 \times 10^{-4}$ units (units defined by Bienroth et al. 1991). The assays in Fig. 1-3 and 5 were performed in the presence of unlabeled yeast RNA, which significantly reduces the activity observed for a fixed amount of CPSF and PAP (not shown). As a result, less CPSF was required in this assay than in the others.
1. Purity. Highly purified CPSF exhibits specificity for U-rich sequences. This is shown in Fig. 5A, in which the preparation of CPSF used in Figures 1-3 was analyzed by SDS-PAGE and silver staining. The three large subunits of CPSF $(160,100$, and $73 \mathrm{kD})$ are the most abundant protein species in this preparation, although other polypeptides are present.

2. Cofractionation. CPSF and the factor conferring U-rich specificity copurify. Peak fractions from each of four successive columns in a CPSF purification were assayed for U-rich specificity, using L1 RNA with and without UUUUUUAU. The specific activity of the U-rich sequence-discriminating factor increased in concert with CPSF during fractionation (not shown). Consistent with these observations, CPSF and the U-rich specificity factor precisely cofractionate. CPSF that had already been partially purified was fractionated by Sephacryl S-500 chromatography. Fractions containing CPSF protein were identified by SDS-PAGE and silver staining (Fig. 5B). Three major bands of $\sim 73,100$, and $160 \mathrm{kD}$, corresponding to CPSF subunits, are present in a peak centered in fraction 64. CPSF activity in each fraction was assayed using a standard substrate for nuclear polyadenylation, adenovirus L3 RNA. As expected, fractions 62-65 contain peak levels of CPSF activity (S. Bienroth and W. Keller, unpubl.). To identify the activity that discriminates the U-rich sequence, we assayed each fraction with c-mos RNA with (Fig. 5C) or without (Fig. 5D) its U-rich element. The distribution of fractions containing polyadenylation activity is virtually identical for the two substrates and coincides with CPSF. Furthermore, in every fraction containing CPSF activity, the presence of a U-rich sequence stimulates polyadenylation.

3. Mobility shift assays. In mobility shift assays using highly purified CPSF, complexes formed on RNAs with and without a U-rich sequence exhibit identical mobility (not shown). These results suggest that complexes formed on RNAs with and without a U-rich sequence do not differ in composition. Furthermore, no U-rich sequence-specific complexes are observed in the absence of AAUAAA (not shown), suggesting that the factor that binds the U-rich element also requires AAUAAA.

CstF, a factor consisting of three polypeptides (Takagaki et al. 1990|, stabilizes complexes between AAUAAA and CPSF in nuclear extracts (Gilmartin and Nevins 1989, 1991; Weiss et al. 1991; Murthy and Manley, 1992). Stabilization by CstF requires sequences downstream of the cleavage site (Gilmartin and Nevins 1989, 1991; Weiss et al. 1991). Because non-AAUAAA sequences are recognized both by CstF and by our CPSF preparations, we determined whether CstF significantly contaminates our purified CPSF. It does not: In Western blotting experiments using an antibody directed against the 64-kD subunit of CstF (a gift of J. Manley, Columbia University, NY| and in cleavage assays in vitro, we detected no CstF in our CPSF preparations (not shown).

From these observations, we conclude that the same factors that are required for nuclear polyadenylationnamely CPSF and PAP-exhibit the sequence specificity seen in the cytoplasm during oocyte maturation.

\section{Frog nuclear polyadenylation factors can display either nuclear or cytoplasmic sequence specificities}

All mRNAs (except certain histone mRNAs) are polyadenylated in the nucleus, yet only those RNAs with U-rich elements receive poly $(A)$ in the cytoplasm during maturation. If CPSF is responsible for poly(A) addition in both compartments, then how do RNAs without a U-rich sequence receive poly(A) in the nucleus? One simple explanation is that the concentration of CPSF might be much higher in the nucleus than in the cytoplasm. If this were true, then the preference of CPSF for U-rich RNAs would be masked in the nucleus: RNAs would be bound by CPSF and polyadenylated regardless of U-rich elements. One prediction of this model is that 
A

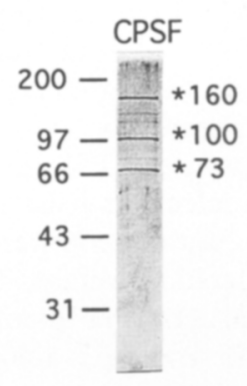

U-rich AAUAAA

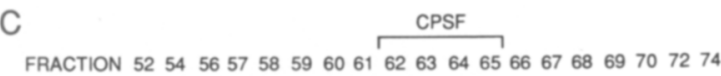

FRACTION 52545657585960616263646566676869707274 $10-$
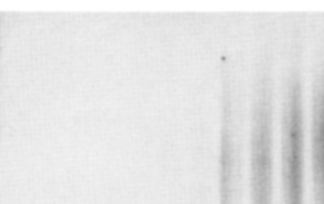

B

FRACTION 52545657585960616263646566676869707274
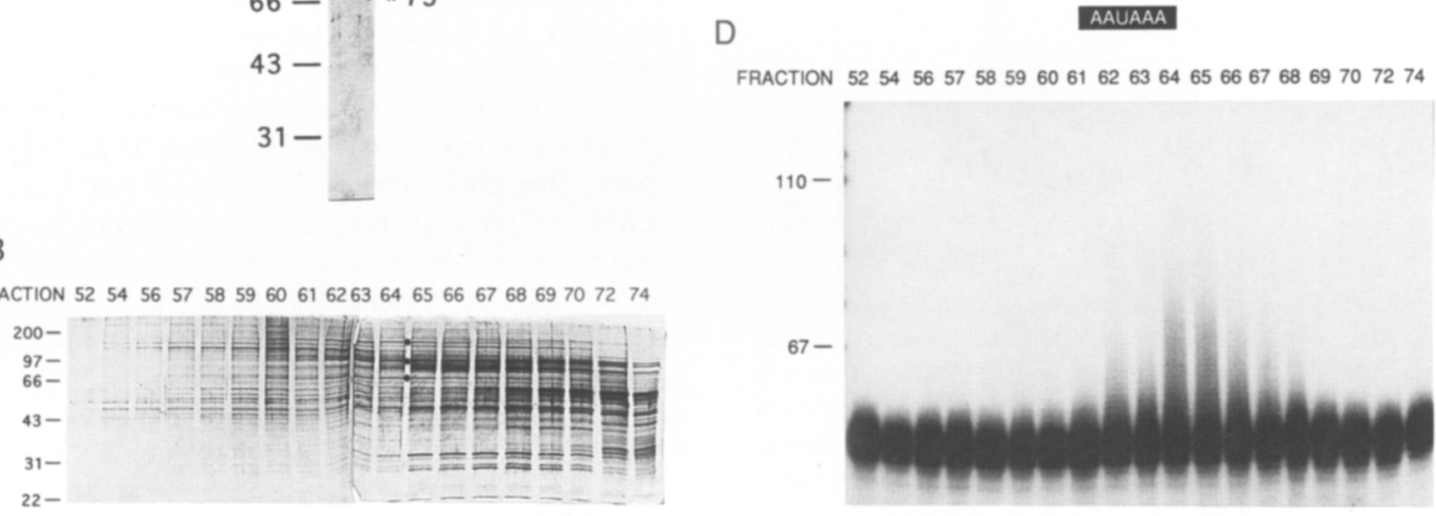

Figure 5. The activity responsible for maturation-type polyadenylation specificity copurifies with CPSF. $(A, B)$ Proteins were analyzed by SDS-PAGE and stained with silver. (A) Approximately 2.5 units of CPSF; $(B) 0.5 \mu$ laliquots of fractions from a Sephacryl S-500 column used to identify CPSF. The S-500 column follows purification by ammonium sulfate precipitation and passage over three columns (see Materials and methods). Proteins that comigrate with the three major subunits of CPSF are marked with an asterisk. $(C, D)$ Bracket indicates peak levels of CPSF activity determined using an adenovirus L3 pre-mRNA (S. Bienroth and W. Keller, unpubl.). One microliter of each S-500 fraction in column buffer (Bienroth et al. 1991) was added to 25 units of purified calf thymus PAP and incubated for $20 \mathrm{~min}$ at $25^{\circ} \mathrm{C}$. $(C)$ Reactions with c-mos wild-type RNA containing both AAUAAA and a U-rich element; $(D)$ reactions with c-mos RNA containing AAUAAA but no U-rich element.

concentrated extracts of frog oocyte nuclei should polyadenylate all RNAs, whereas dilute extracts should polyadenylate only those RNAs with U-rich elements.

To test this prediction, we prepared microscale nuclear extracts and examined their sequence specificity when concentrated or dilute (Fig. 6). Frog oocytes were enucleated under mineral oil to prevent leakage of nuclear contents, and the isolated nuclei were combined. To prepare concentrated reactions, nuclei were diluted only 2 -fold by the addition of labeled RNA and buffer; to prepare dilute reactions, nuclei were diluted 20 -fold. Reactions were incubated under mineral oil and polyadenylation was assayed by gel electrophoresis.

In concentrated nuclear extracts (Fig. 6, lanes 3,4), RNAs with and without UUUUUUAU both receive poly(A) efficiently: The tail added is very long, and most of the substrate reacts. In dilute reactions (Fig. 6, lanes $5,6)$, the UUUUUUAU sequence stimulates polyadenylation dramatically. Less of the substrate receives poly(A), and the tails are shorter, as predicted. Oligo(dT)/RNase
$\mathrm{H}$ digestion confirmed that the lengthening seen in both concentrated and dilute reactions is caused by the addition of a poly(A) tail (not shown). We conclude that frog nuclei contain factors capable of displaying either nuclear or cytoplasmic specificity, depending on their concentration.

\section{Discussion}

Our results demonstrate that polyadenylation by purified calf thymus CPSF and PAP in vitro exhibits the same sequence specificity as does polyadenylation in the cytoplasm during oocyte maturation. Polyadenylation by these factors is markedly stimulated by the presence of a U-rich element near AAUAAA. The enhanced polyadenylation of RNAs containing U-rich elements is caused by their elevated affinity for CPSF. Addition of CPSF stimulates polyadenylation in oocyte extracts, implying that a CPSF-like factor may be rate-limiting before maturation. Sequences upstream of AAUAAA, by 


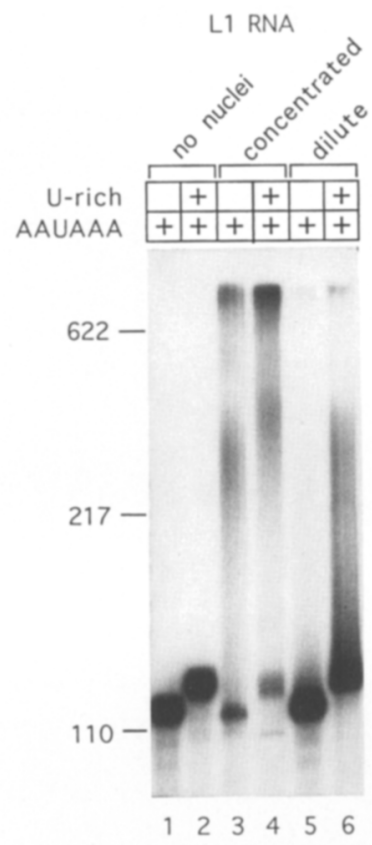

Figure 6. Frog nuclear polyadenylation factors can display both nuclear and cytoplasmic polyadenylation specificities. RNAs were incubated with frog oocyte nuclei under concentrated or dilute conditions for $25 \mathrm{~min}$ at $25^{\circ} \mathrm{C}$. The substrates used are the L1 RNAs shown in Fig. 1B. (Lanes 1,3,5) Wild-type L1 RNA; (lanes 2,4,6) L1 with a UUUUUUAU insertion. (Lanes $1,2)$ No nuclei; (lanes 3,4) concentrated nuclear reactions; (lanes $5,6)$ dilute nuclear reactions.

enhancing CPSF binding, may not only stimulate polyadenylation in the cytoplasm but may also enhance cleavage and polyadenylation of certain pre-mRNAs in the nucleus.

We propose that CPSF and PAP catalyze both nuclear and cytoplasmic polyadenylation, and that CPSF imparts sequence specificity to both reactions. In the cytoplasm, we suggest that CPSF is limiting, such that only RNAs with U-rich elements are polyadenylated efficiently. In the nucleus, where polyadenylation factors are abundant, all AAUAAA-containing RNAs are polyadenylated. Differences in the concentration of CPSF, PAP, or RNA could result in competition for polyadenylation factors in one compartment but not the other. Consistent with this model, polyadenylation factors in the frog nucleus can display either nuclear or cytoplasmic polyadenylation specificities, depending on their concentration (Fig. 6).

In the nucleus, the interaction between CPSF and AAUAAA may be enhanced by other proteins. Nuclear pre-mRNAs associate with CPSF, PAP, and a collection of other trans-acting factors to form a large complex in which cleavage and polyadenylation then occur (for review, see Wahle and Keller 1992). CstF, which enhances cleavage in vitro, does so by stabilizing the interaction between CPSF and AAUAAA in these precleavage complexes (Gilmartin and Nevins 1989, 1991; Weiss et al. 1991; Murthy and Manley 1992) and, therefore, may also contribute to the difference in CPSF binding in nucleus and cytoplasm.

An obvious prediction of our model is that PAP and CPSF are located both in the nucleus and the cytoplasm. Preliminary results indicate that CPSF is present in both compartments (A. Bilger, A. Jenny, W. Keller, and M. Wickens, unpubl.). Partitioning between the two compartments might be controlled by regulated modification, such as the phosphorylation and dephosphorylation reactions that govern the distribution of SWI5 (Moll et al. 1991), or by interaction with other proteins, as in the regulation of NF- $\kappa$ B nuclear import by IF- $\kappa \mathrm{B}$ (for review, see Baeuerle 1991). Alternatively, nuclear and cytoplasmic factors might be related but non-identical, such that they partition differently.

The preparations of CPSF and PAP used in this report probably are nuclear, at least in part. Both factors are nearly identical to their counterparts purified from HeLa nuclei, in molecular weight, fractionation properties, and biochemical activities (Bienroth et al. 1991). Furthermore, the CPSF and PAP that we have used support mRNA $3^{\prime}$ end cleavage, an exclusively nuclear event (Murthy and Manley 1992; K. Beyer, E. Wahle, and W. Keller, unpubl.). However, somatic cells may contain cytoplasmic CPSF and PAP, consistent with reports of PAP activity in cytoplasmic fractions (Ryner et al. 1989; Edmonds 1992) and of possible cytoplasmic increases in poly(A) tail length (e.g., Muschel et al. 1986; Paek and Axel 1987; Robinson et al. 1988; for review, see Brawerman 1981).

Our results strongly suggest that CPSF itself recognizes both U-rich elements and AAUAAA. Other observations are consistent with the notion that a single factor recognizes both sequences. The activities that bind to these two sequences are, as yet, chromatographically inseparable (C. Fox, A. Bilger, and M. Wickens, unpubl.). Mutations in either the CPE or in AAUAAA greatly decrease or eliminate formation of specific polyadenylation complexes in egg extracts (Paris and Richter 1990). Finally, an excess of either of these sequence elements alone does not prevent complex formation or polyadenylation of RNAs containing both sequences (Paris and Richter 1990).

The onset of polyadenylation during maturation may be caused by the activation or expression of a CPSF-like factor. Calf thymus CPSF, like the egg RNA-binding fraction, activates polyadenylation in extracts prepared from oocytes before maturation. This suggests that a CPSF-like molecule is limiting before maturation and may be synthesized or activated to turn on polyadenylation during oocyte maturation.

\section{Extension of $\operatorname{poly}(A)$ tails}

The apparent mechanism of poly(A) tail extension differs in the nucleus and the cytoplasm. In the nucleus, AAUAAA is required only to add the first 10 adenosines; after that point, extension of the tail is sequence-independent (Sheets and Wickens 1989). In the cytoplasm, however, AAUAAA and a CPE are required to extend 
poly(A) tails that already are 25-75 nucleotides long. This apparent difference in sequence specificity may be only superficial. A sequence-independent poly(A) extension activity might be active in the cytoplasm, as it is in the nucleus, but be fully counteracted by the poly(A)removing activity that is activated during maturation (Fox and Wickens 1990; Varnum and Wormington 1990). In this view, CPEs would accelerate the rate of tail extension and therefore result in a net increase in poly(A) length during maturation.

The length of poly(A) added to different mRNAs in the cytoplasm is highly controlled. For example, whereas cyclin B1 mRNA receives $\sim 300$ adenosines during maturation, c-mos mRNA receives only 75 (Sheets et al. 1994). This control of final poly(A) length is reconstituted in crude egg extracts and in mixtures of the RNAbinding and PAP activities of the egg (Paris and Richter 1990; Fox et al. 1992; Sheets et al. 1994), but is missing in reactions containing only purified CPSF and PAP /this paper). These results imply that an additional factor, present in oocyte and egg extracts, is required to regulate tail length during maturation. Such a factor might be related to PABII, which promotes poly(A) tail elongation in the nucleus and causes it to terminate after 250 adenosines have been added (Wahle 1991b). However, unlike PABII, the cytoplasmic length-controlling factors must cause a different length of poly $(A)$ to be added to each mRNA.

\section{Implications for mRNA 3' end formation in the nucleus}

In the nucleus, formation of the $3^{\prime}$ terminus of certain mRNAs, such as those of human immunodeficiency virus (HIV) and other viruses, requires sequences upstream of AAUAAA (e.g., Gilmartin et al. 1992; Schek et al. 1992; for review, see Imperiale and DeZazzo 1991). These upstream sequences may, like CPEs, enhance the interaction of CPSF with AAUAAA. Pre-mRNAs may require additional sequences for cleavage either because their AAUAAA sequence is noncanonical (e.g., UAUAAA) or because it is obscured by secondary structure. Mutation of the hepatitis $B$ virus from its natural UAUAAA sequence to AAUAAA decreases the requirement for upstream elements (Russnak and Ganem 1990). Thus CPEs and nuclear upstream elements may be functionally, and perhaps structurally, related.

In pre-mRNAs containing more than one potential polyadenylation site, the choice between sites may be regulated by CstF concentration (Gilmartin and Nevins 1989, 1991; Weiss et al. 1991). CstF stabilizes complexes between AAUAAA and CPSF, in a reaction that requires sequences downstream of the cleavage site. At low CstF concentrations, only sites with "high affinity" downstream sequences are used in vitro. These same arguments apply, a priori, to CPSF itself: at low CPSF concentrations, only those RNAs that contain additional sequences near AAUAAA, such as a CPE, may be cleaved.

In summary, sequences that enhance CPSF binding may stimulate mRNA 3 '- end formation in the nucleus, as well as polyadenylation in the cytoplasm. The term "cytoplasmic polyadenylation element" should not be taken to imply an exclusively cytoplasmic function.

\section{A core polyadenylation apparatus in the cytoplasm}

CPSF and PAP may constitute a core polyadenylation apparatus that acts on many, if not all, of the mRNAs that receive poly(A) during maturation and early development. Other factors might modulate the activity of this core apparatus, determining, for example, when poly $(\mathrm{A})$ is added, or how long the tail becomes. In this view, cytoplasmic polyadenylation is analogous to transcription in that the activity of a single core apparatus is modulated by sequence-specific regulatory factors. As in the interaction of RNA polymerase with promoter elements, the intrinsic strength of the interaction between CPSF and sequences near AAUAAA may determine the basal efficiency of the reaction.

Proteins of 58 and $82 \mathrm{kD}$ have been cross-linked by UV light to CPE-containing RNAs (McGrew and Richter 1990; Paris et al. 1991|. Each of these proteins has been detected on a different mRNA. These and related observations suggest a role for these proteins in polyadenylation. The 58- and $82-\mathrm{kD}$ proteins could promote polyadenylation by binding to different CPEs, without the involvement of CPSF. Alternatively, binding of the 58and $82-\mathrm{kD}$ proteins could enhance, prevent, or delay binding of CPSF to specific mRNAs. It is also possible that one or both of these proteins binds CPE-containing RNAs generally but only cross-links efficiently with certain RNAs. Such an explanation would be consistent with these proteins being part of a core polyadenylation apparatus containing CPSF and PAP.

Negative regulatory elements have been identified genetically in the 3' UTRs of several mRNAs that are critical in early development in Drosophila and Caenorhabditis elegans, including bicoid, tra-2, and fem-3 (Ahringer and Kimble 1991; Wharton and Struhl 1991; Gavis and Lehmann 1992; Goodwin et al. 1993). Mutations in some of these elements have been shown to increase poly(A) tail length, as well as translational activity. Similarly, negative elements in the $3^{\prime}$ UTR of frog $\mathrm{Cl} 2$ mRNA specifically prevent poly(A) addition until after fertilization (Simon et al. 1992). Factors interacting with such negative elements might repress a core, cytoplasmic polyadenylation apparatus.

\section{Materials and methods}

RNA substrates

The number +1 designates the last nucleotide in the mRNA before the poly(A) tail. Thus, $-50 /+1 \mathrm{c}-$ mos contains the last 50 nucleotides of c-mos mRNA (Fox et al. 1992). In every case except $-58 /+1$ H4 RNA, the last nucleotide of the transcript is the poly(A) site of the mRNA.

$-50 /+1 c-\operatorname{mos} R N A$ and $-50 /+1 c-\operatorname{mos} R N A$ containing $a$ UUUUAU substitution or a point mutation in AAUAAA Oligonucleotides carrying c-mos and $\mathrm{T} 7$ promoter sequences 
were annealed, as described by Fox et al. (1992). Single-stranded regions were filled in with T4 DNA polymerase, and the resulting blunt-ended fragments were ligated to filled-in EcoRI-cut pSP64 (Promega) to yield p-50/+1c-mos, p-50/+1c-mos: Usub, and p-50/+1c-mos:AAGAAA. To generate the 50-nucleotide transcripts, plasmids were digested with $X b a \mathrm{I}$ and incubated with T7 RNA polymerase (sequences in Fig. 1A). The plasmid-derived RNA containing the UUUUAU substitution (a 49-nucleotide transcript) lacks a single nucleotide downstream of AAUAAA. The deletion does not affect the activity of the RNA (C. Fox and M. Wickens, unpubl.).

-101/+1 ribosomal protein L1 RNAs - 101/+1 L1 RNA (a 114-nucleotide transcript) was generated as described (Fox et al. 1992) from pLl3z. A pGEM3Z (Promega)-derived plasmid encoding - 101/ + 1 L1 RNA with UUUUUUAU (pL13zmut) was generated by site-directed mutagenesis using the polymerase chain reaction. The UUUUUUAU is inserted 8 nucleotides upstream of AAUAAA (sequences in Fig. 1B). The plasmid was cut with AfIII and incubated with T7 RNA polymerase to yield L1 RNA containing UUUUUUAU (a 122-nucleotide transcript).

$-80 /+1$ Xfin RNA By annealing complementary oligonucleotides, the terminal 80 nucleotides of an Xfin cDNA were cloned into pGEM3Z, flanked by EcoRI and NsiI-BamHI sites at the $5^{\prime}$ and $3^{\prime}$ ends, respectively. (In cloning, a single nucleotide 73 nucleotides from the $3^{\prime}$ end was deleted.) To generate -80 / +1 Xfin RNA (a 90-nucleotide transcript), this plasmid (pXfin3Z) was digested with NsiI and incubated with T7 RNA polymerase. As expected (Ruiz i Altaba et al. 1987; Fox and Wickens 1990), $-80 /+1$ Xfin RNA injected into oocytes does not receive poly $\mid A)$ during maturation when injected (A. Bilger, D. Daniel, and M. Wickens, unpubl.).

$-58 /+1$ H4 RNA This 95-nucleotide RNA was generated as described (Fox and Wickens 1990).

$-83 /+2$ cyclin B1 RNA This 94-nucleotide RNA was generated as described (Sheets et al. 1994).

\section{Transcription in vitro}

RNAs were prepared using T7 or SP6 RNA polymerase as described by Fox et al. (1989), except that RNA pellets were resuspended in water, not in $88 \mathrm{~mm} \mathrm{NaCl}$. RNAs had specific activities of $2 \times 10^{3}$ to $10 \times 10^{3} \mathrm{cpm} / \mathrm{fmole}$.

Preparation of Xenopus laevis oocyte and egg extracts and fractions

Crude frog oocyte extracts were prepared as described (Fox et al. 1992). From frog egg extract, separate fractions containing the PAP and RNA-binding activities were obtained by elution from DEAE-Sepharose with 0.1 and $0.3 \mathrm{M} \mathrm{KCl}$, respectively (Fox et al. 1992).

\section{Preparation of purified calf thymus polyadenylation factors}

The CPSF used in Figures 1-3 was purified as described (Bienroth et al. 1991). For the preparation used in Figure 4, the spermine-agarose column was omitted. The preparation of CPSF used in Figure 5 was obtained using the following scheme: DEAE-Sepharose, blue-Sepharose, $80 \%$ ammonium sulfate, heparin-Sepharose, $70 \%$ ammonium sulfate, S-500 gel filtration, Mono $\mathrm{Q}$, poly $(\mathrm{A})-$ Sepharose, and heparin-Sepharose to concentrate. Note that the $\mathrm{pH}$ of the purification buffers in
Bienroth et al. was given incorrectly as 7.0; the pH should be 7.9. Units of CPSF are as defined by Bienroth et al. (1991). These units differ from those used by Fox et al. (1992).

PAP was purified as described (Wahle, 1991a).

\section{Polyadenylation assays}

Sequence-specific polyadenylation was assayed in 9- $\mu$ l reactions containing $6 \mu \mathrm{l}$ of protein in buffer $\mathrm{B}[100 \mathrm{mM} \mathrm{KCl}, 50 \mathrm{~mm}$ Tris, $150 \mu \mathrm{M}$ EDTA, $10 \%$ glycerol $\left(\mathrm{pH} 8.5\right.$, at $\left.\left.5^{\circ} \mathrm{C}\right)\right], 1-2$ fmoles of RNA, $2.2 \mathrm{U} / \mu$ l of RNasin (Promega), $2.8 \mathrm{~mm} \mathrm{DTT,} 0.7 \mu \mathrm{g} / \mu \mathrm{l}$ of yeast RNA (Sigma), $1.1 \mathrm{~mm} \mathrm{MgCl}_{2}, 1.1 \mathrm{~mm}$ ATP, $0.11 \mathrm{~mm}$ EGTA ( $\mathrm{pH} 7.7$ ), and $8.3 \mathrm{mM}$ creatine phosphate. Assay mixtures were assembled on ice, and incubated at $25^{\circ} \mathrm{C}$ for $20 \mathrm{~min}$. RNAs were cleaned using phenol/chloroform extraction and precipitation with ethanol, and analyzed by gel electrophoresis through a denaturing $6 \%$ or $8 \%$ polyacrylamide gel followed by autoradiography.

Nonspecific polyadenylation activity (i.e., PAP activity in the absence of any other factors) was assayed in the presence of $\mathrm{Mn}^{2^{+}}$, as described (Wahle 1991a). A unit of nonspecific polymerase activity is defined here as 1 pmole of ATP incorporated per 10-min incubation (Fox et al. 1992).

\section{SDS-PAGE analysis of purified CPSF}

Fully and partially purified CPSF was boiled for $\sim 5 \mathrm{~min}$ in protein gel loading buffer ( $1 \times$ stacking gel buffer, $20 \%$ glycerol, $0.025 \%$ bromophenol blue, $1.43 \mathrm{~m} \beta$-mercaptoethanol, $6 \%$ SDS and analyzed by electrophoresis through a $4.2 \%$ (stacking) $/ 9 \%$ (separation) polyacrylamide gel (Laemmli 1970). Proteins were identified by silver staining.

\section{Analysis of CPSF RNA complexes}

To analyze complexes formed between CPSF and labeled RNAs (Fig. 4), incubations were carried out in $10 \%$ glycerol, $2.6 \%$ polyvinyl alcohol, $25 \mathrm{~mm}$ Tris- $\mathrm{HCl}(\mathrm{pH} 7.9), 50 \mathrm{mM} \mathrm{KCl}, 2 \mathrm{mM}$ $\mathrm{MgCl}_{2}, 1 \mathrm{mM}$ DTT, $0.05 \mathrm{mM}$ EDTA, $0.01 \% \mathrm{NP}-40,0.4 \mathrm{mg} / \mathrm{ml}$ of methylated BSA, and 0.5 units of RNAguard (Pharmacia). RNA (65 fmoles of each) and the amount of CPSF indicated were combined. Reactions $(25 \mu \mathrm{l})$ were incubated for $10 \mathrm{~min}$ at room temperature. Complete reaction mixtures were loaded onto a $4 \%$ nondenaturing gel already running at $250 \mathrm{~V}$. After electrophoresis, RNAs were transferred from the gel to NA45 paper (Schleicher \& Schuell) and visualized by autoradiography.

Complex and free RNA bands were excised after autoradiography, and RNAs were eluted as described (Keller et al. 1991). Eluted RNAs were purified by phenol/chloroform extraction, precipitated with ethanol, and then analyzed by electrophoresis under denaturing conditions, followed by autoradiography.

Relative dissociation constants were calculated according to the following equation (see Weeks and Crothers 1991):

$$
K_{\text {rel }}=K_{\mathrm{d}}(\mathrm{AC}) / K_{\mathrm{d}}(\mathrm{BC})=\left[A_{\text {free }}\right][\mathrm{BC}] /\left[B_{\text {free }}\right][A C]
$$

\section{Mini-extracts of oocyte nuclei}

Individual frog oocytes were placed in a petri dish under light mineral oil (Sigma). The oocytes were then pricked with a fine injection needle at the animal pole and gently squeezed with forceps until the nucleus emerged. The needle was then used to gently separate the nucleus from the oocyte cytoplasm. Nuclei bore minor cytoplasm contamination. For each reaction, $10 \mathrm{nu}-$ clei $(\sim 0.5 \mu 1$ total) were gathered under mineral oil into one cluster in the dish. Polyadenylation was assayed in the presence 
of the following: $1.4 \mathrm{fmole} / \mu \mathrm{l}$ of labeled RNA, $1 \mathrm{U} / \mu \mathrm{l}$ of RNasin (Promega), $1.25 \mathrm{~mm}$ DTT, $0.5 \mathrm{~mm} \mathrm{MgCl} \mathrm{Mg}_{2}, 0.5 \mathrm{~mm}$ ATP, $0.05 \mathrm{~mm}$ EGTA (pH 7.7), and $3.75 \mathrm{~mm}$ creatine phosphate. Concentrated reactions were brought to $1 \mu \mathrm{l}$ by the addition of $0.25 \mu \mathrm{l}$ of buffer $B$; dilute reactions were brought to $20 \mu$ l by the addition of $14.5 \mu \mathrm{l}$ of the same buffer. Nuclei and exogenous reaction components were mixed thoroughly with a needle, which breaks the nuclei, and incubated for $25 \mathrm{~min}$ at $25^{\circ} \mathrm{C}$.

\section{Molecular weight standards}

MspI fragments of pBR322 were labeled using the Klenow fragment of DNA polymerase I and $\left[\alpha^{32} \mathrm{P}\right] \mathrm{dCTP}$ (Amersham or DuPont). Protein standards were "Rainbow" (Amersham) and "Low Range" (Bio-Rad).

\section{Acknowledgments}

We are very grateful to Silke Bienroth and Walter Keller for CPSF, to Sunnie Thompson and Mike Sheets for plasmids, and to Don Daniel for injections. We especially thank Scott Ballantyne and Wayne Forrester for thoughtful discussions, and we thank other members of the Wickens laboratory for helpful suggestions and comments on the manuscript. We appreciate the excellent assistance of Laura VanderPloeg and Adam Steinberg in assembling figures. This work was supported by a research grant (GM31892) and research career development award (GM00521) to M.W., a National Science Foundation predoctoral fellowship to A.B., a Ford Foundation Fellowship to C.F., and grants from the Kantons of Basel and the Schweizerisches $\mathrm{Na}$ tionalfonds to E.W.

The publication costs of this article were defrayed in part by payment of page charges. This article must therefore be hereby marked "advertisement" in accordance with 18 USC section 1734 solely to indicate this fact.

\section{References}

Ahringer, J. and J. Kimble. 1991. Control of the sperm-oocyte switch in Caenorhabditis elegans hermaphrodites by the fem-3 3' untranslated region. Nature 349: 246-248.

Bardwell, V.J., D. Zarkower, M. Edmonds, and M. Wickens. 1990. The enzyme that adds poly(A) to mRNAs is a classical PAP. Mol. Cell. Biol. 10: 846-849.

Bardwell, V.J., M. Wickens, S. Bienroth, W. Keller, B.S. Sproat, and A.I. Lamond. 1991. Site-directed ribose methylation identifies $2 '-\mathrm{OH}$ groups in polyadenylation substrates critical for AAUAAA recognition and poly(A) addition. Cell 65: 125-133.

Baeuerle, P.A. 1991. The inducible transcription activator NFКB: Regulation by distinct protein subunits. Biochim. Biophys. Acta 1072: 63-80.

Bienroth, S., E. Wahle, C. Suter-Crazzolara, and W. Keller. 1991. Purification of the cleavage and polyadenylation factor involved in the 3 '-processing of messenger RNA precursors. $J$. Biol. Chem. 266: 19768-19776.

Brawerman, G. 1981. The role of the poly(A) sequence in mammalian messenger RNA. CRC Crit. Rev. Biochem. 10: 1-38.

Conway, L. and M. Wickens. 1987. Analysis of mRNA $3^{\prime}$ end formation by modification interference: The only modifications which prevent processing lie in AAUAAA and the poly(A) site. EMBO I. 6: 4177-4184.

Dworkin, M.B. and E. Dworkin-Rastl. 1985. Changes in RNA titers and polyadenylation during oogenesis and oocyte mat uration in Xenopus laevis. Dev. Biol. 112: 451-457.

- 1990. Functions of maternal mRNA in early development. Mol. Reprod. Dev. 26: 261-297.

Edmonds, M. 1990. Polyadenylate polymerases. Methods Enzymol. 181: 161-170.

Fox, C.A. and M. Wickens. 1990. Poly(A) removal during oocyte maturation: A default reaction selectively prevented by specific sequences in the $3^{\prime}$ UTR of certain maternal mRNAs. Genes \& Dev. 4: 2287-2298.

Fox, C.A., M.D. Sheets, and M.P. Wickens. 1989. Poly(A) addition during maturation of frog oocytes: Distinct nuclear and cytoplasmic activities and regulation by the sequence UUUUUAU. Genes \& Dev. 3: 2151-2162.

Fox, C.A., M.D. Sheets, E. Wahle, and M. Wickens. 1992. Polyadenylation of maternal mRNA during oocyte maturation: Poly $(\mathrm{A})$ addition in vitro requires a regulated RNA-binding activity and a poly(A) polymerase. $E M B O /$. 11: 5021-5032.

Gavis, E.R. and R. Lehmann. 1992. Localization of nanos RNA controls embryonic polarity. Cell 71: 301-313.

Gilmartin, G.M. and J.R. Nevins. 1989. An ordered pathway of assembly of components required for polyadenylation site recognition and processing. Genes \& Dev. 3: 2180-2189.

- 1991. Molecular analyses of two poly(A) site-processing factors that determine the recognition and efficiency of cleavage of the pre-mRNA. Mol. Cell. Biol. 11: 2432-2438.

Gilmartin, G.M., E.S. Fleming, and J. Oetjen. 1992. Activation of HIV-1 pre-mRNA $3^{\prime}$ processing in vitro requires both an upstream element and TAR. EMBO J. 11: 4419-4428.

Goodwin, E.B., P.G. Okkema, T.C. Evans, and J. Kimble. 1993. Translational regulation of tra-2 by its $3^{\prime}$ untranslated region controls sexual identity in C. elegans. Cell 75: 329-339.

Huarte, J., A. Stutz, M.L. O'Connell, P. Gubler, D. Belin, A.L. Darrow, S. Strickland, and J.-D. Vassalli. 1992. Transient translational silencing by reversible mRNA deadenylation. Cell 69: 1021-1030.

Imperiale, M.J. and J.D. DeZazzo. 1991. Poly(A) site choice in retroelements: Deja vu all over again? New Biol. 3: 531-537.

Jackson, R.J. and N. Standart. 1990. Do the poly(A) tail and 3' untranslated region control mRNA translation? Cell 62: 1524.

Keller, W., S. Bienroth, K.M. Lang, and G. Christofori. 1991. Cleavage and polyadenylation factor $\mathrm{CPF}$ specifically interacts with the pre-mRNA $3^{\prime}$ processing signal AAUAAA. EMBO \%. 10: 4241-4249.

Laemmli, U.K. 1970. Cleavage of structural proteins during the assembly of the head of bacteriophage T4. Nature 227: 680685.

McGrew, L.L. and J.D. Richter. 1990. Translational control by cytoplasmic polyadenylation during Xenopus oocyte maturation: Characterization of cis and trans elements and regulation by cyclin/MPF. EMBO I. 9: 3743-3751.

McGrew, L.L., E. Dworkin-Rastl, M.B. Dworkin, and J.D. Richter. 1989. Poly(A) elongation during Xenopus oocyte maturation is required for translational recruitment and is mediated by a short sequence element. Genes \& Dev. 3: 803-815.

Moll, T., G. Tebb, U. Surana, H. Rabitsch, and K. Nasmyth. 1991. The role of phosphorylation and the CDC28 protein kinase in cell cycle-regulated nuclear import of the S. cerevisiae transcription factor SWI5. Cell 66: 743-758.

Murthy, K.G. and J.L. Manley. 1992. Characterization of the multisubunit cleavage-polyadenylation specificity factor from calf thymus. J. Biol. Chem. 267: 14804-14811.

Muschel, R., G. Khoury, and L.M. Reid. 1986. Regulation of insulin mRNA abundance and adenylation: Dependence on hormones and matrix substrata. Mol. Cell. Biol. 6: 337-341. 
Paek, I. and R. Axel. 1987. Glucocorticoids enhance stability of human growth hormone mRNA. Mol. Cell. Biol. 7: 14961507.

Paris, J. and M. Philippe. 1990. Poly(A) metabolism and polysomal recruitment of maternal mRNAs during early Xenopus development. Dev. Biol. 140: 221-224.

Paris, J. and J.D. Richter. 1990. Maturation-specific polyadenylation and translational control: Diversity of cytoplasmic polyadenylation elements, influence of poly(A) tail size, and formation of stable polyadenylation complexes. Mol. Cell. Biol. 10: 5634-5645.

Paris, J., K. Swenson, H. Piwnica-Worms, and J.D. Richter. 1991. Maturation-specific polyadenylation: In vitro activation by p34 ${ }^{c d c 2}$ and phosphorylation of a $58-\mathrm{kD}$ CPE-binding protein. Genes \& Dev. 5: 1697-1708.

Robinson, B.G., D.M. Frim, W.J. Schwartz, and J.A. Majzoub. 1988. Vasopressin mRNA in the suprachiasmatic nuclei: Daily regulation of polyadenylate tail length. Science 241: 342-344.

Ruiz i Altaba, A., H. Perry-O'Keefe, and D.A. Melton. 1987. Xfin: An embryonic gene encoding a multifingered protein in Xenopus. EMBO I. 6: 3065-3070.

Russnak, R. and D. Ganem. 1990. Sequences $5^{\prime}$ to the polyadenylation signal mediate differential poly(A) site use in hepatitis B viruses. Genes \& Dev. 4: 764-776.

Ryner, L.C., Y. Takagaki, and J.L. Manley. 1989. Multiple forms of PAPs purified from HeLa cells function in specific mRNA 3'-end formation. Mol. Cell. Biol. 9: 4229-4238.

Schek, N., C. Cooke, and J.C. Alwine. 1992. Definition of the upstream efficiency element of the simian virus 40 late poly. adenylation signal by using in vitro analyses. Mol. Cell. Biol. 12: 5386-5393.

Sheets, M.D. and M. Wickens. 1989. Two phases in the addition of a poly(A) tail. Genes \& Dev. 3: 1401-1412.

Sheets, M.D., C.A. Fox, T. Hunt, G. Vande Woude, and M. Wickens. 1994. The 3' UTRs of c-mos and cyclin mRNAs control translation by regulating cytoplasmic polyadenylation. Genes \& Dev. 8: 926-938.

Simon, R., J-P. Tassan, and J.D. Richter. 1992. Translational control by poly(A) elongation during Xenopus development: Differential repression and enhancement by a novel cytoplasmic polyadenylation element. Genes \& Dev. 6: 25802591.

Takagaki, Y., L.C. Ryner, and J.L. Manley. 1988. Separation and characterization of a poly(A) polymerase and a cleavage/ specificity factor required for pre-mRNA polyadenylation. Cell 52: 731-742.

Takagaki, Y., J.L. Manley, C.C. MacDonald, J. Wilusz, and T. Shenk. 1990. A multisubunit factor, CstF, is required for polyadenylation of mammalian pre-mRNAs. Genes \& Dev. 4: $2112-2120$.

Varnum, S.M. and M.W. Wormington. 1990. Deadenylation of maternal mRNAs during Xenopus oocyte maturation does not require specific cis-sequences: A default mechanism for translational control. Genes \& Dev. 4: 2278-2286.

Wahle, E. 1991a. Purification and characterization of a mammalian polyadenylate polymerase involved in the 3 ' end processing of messenger RNA precursors. J. Biol. Chem. 266: 3131-3139.

- $1991 \mathrm{~b}$. A novel poly(A) binding protein acts as a specificity factor in the second phase of messenger RNA polyadenylation. Cell 66: 759-768.

Wahle, E. and W. Keller. 1992. The biochemistry of 3'-end cleavage and polyadenylation of messenger RNA precursors. Annu. Rev. Biochem. 61: 419-440.

Wahle, E., G. Martin, E. Schlitz, and W. Keller. 1991. Isolation and expression of cDNA clones encoding mammalian poly(A) polymerase. EMBO J. 10: 4251-4257.

Weeks, K.M. and D.M. Crothers. 1991. RNA recognition by tat-derived peptides: Interaction in the major groove? Cell 66: $577-588$.

Weiss, E.A., G.M. Gilmartin, and J.R. Nevins. 1991. Poly(A) site efficiency reflects the stability of complex formation involving the downstream element. EMBO J. 10: 215-219.

Wharton, R.P. and G. Struhl. 1991. RNA regulatory elements mediate control of Drosophila body pattern by the posterior morphogen nanos. Cell 67: 955-967.

Wickens, M. 1992. Forward, backward, how much, when: Mechanisms of poly(A) addition and removal and their role in early development. Sem. Dev. Biol. 3: 399-412.

Wigley, P.L., M.D. Sheets, D.A. Zarkower, M.E. Whitmer, and M. Wickens. 1990. Polyadenylation of mRNA: Minimal substrates and a requirement for the $2^{\prime}$ hydroxyl of the $U$ in AAUAAA. Mol. Cell. Biol. 10: 1705-1713. 


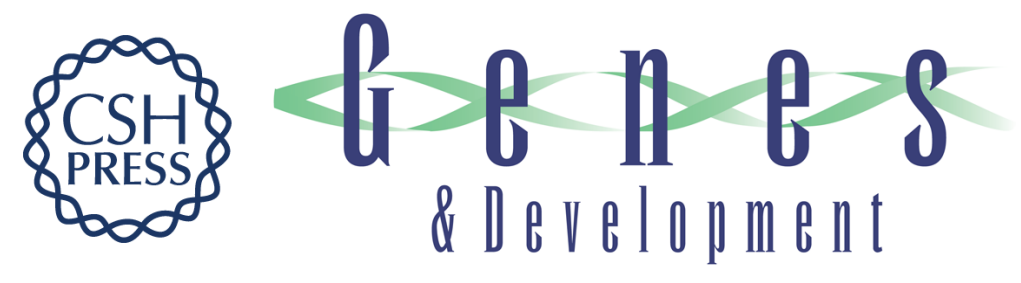

\section{Nuclear polyadenylation factors recognize cytoplasmic polyadenylation elements.}

A Bilger, C A Fox, E Wahle, et al.

Genes Dev. 1994, 8:

Access the most recent version at doi:10.1101/gad.8.9.1106

References This article cites 52 articles, 23 of which can be accessed free at: http://genesdev.cshlp.org/content/8/9/1106.full.html\#ref-list-1

License

Email Alerting

Receive free email alerts when new articles cite this article - sign up in the box at the top Service right corner of the article or click here.

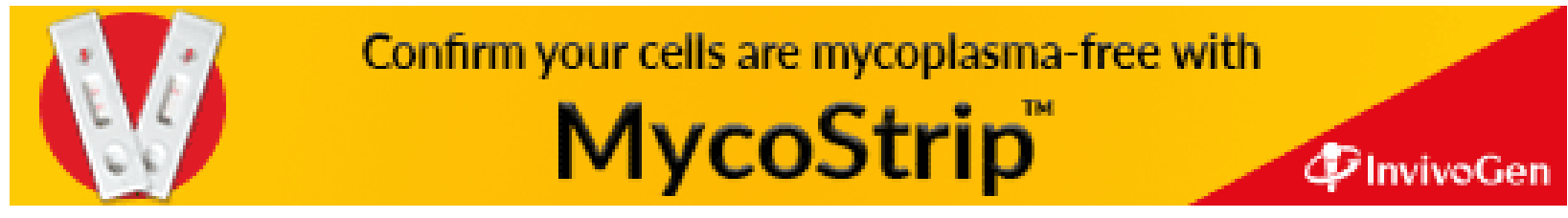

